\title{
La evaluación de competencias en titulaciones Jurídico-Laborales
}

The assessment of competences in legal-labour qualifications

\author{
Dra. Ana Isabel PÉREZ CAMPOS \\ Universidad Rey Juan Carlos. Madrid
}

\begin{abstract}
Resumen: El modelo de evaluación centrado en la adquisición de competencias genéricas y específicas representa uno de los cambios más significativos del Espacio Europeo de Educación Superior. El profesorado universitario se enfrenta con un conjunto de competencias, excesivamente amplio, cuya definición entiende con dificultades, pidiéndosele al tiempo que diseñe aquellas metodologías docentes, actividades formativas y sistemas de evaluación que posibiliten a los alumnos la adquisición de tales competencias, en los denominados resultados de aprendizaje.
\end{abstract}

Este estudio tiene como objetivo prioritario el tratar de determinar cómo han de valorarse y evaluarse las competencias adquiridas por los alumnos en titulaciones jurídico-laborales. Y, más específicamente a dilucidar si los resultados conseguidos en los últimos años, con la modificación de las metodologías docentes y los sistemas de evaluación han sido realmente tan eficaces como se esperaba.

\footnotetext{
Abstract: The evaluation model focused on the acquisition of generic and specific skills represents one of the most significant changes in the European Higher Education Area. The university faculty is faced with a set of competencies, excessively broad, whose definition understands with difficulties, asking at the same time to design those methodologies, technologies and means of evaluation that enable students to acquire such skills in the so-called learning outcomes.

This study has as a priority objective to try to determine how the skills acquired by students in legal-labor qualifications should be assessed. And, more specifically to elucidate whether the results achieved in a few years with the modification of teaching methodologies have been really as positive as expected.
} 
Palabras Clave: Educación Superior; Innovación metodológica; Evaluación Competencias; Titulaciones jurídicas.

Keywords: Higher education, innovation methodological, assessment of competences, qualifications.

\section{Sumario:}

I. Introducción.

II. Evaluación de competencias.

2.1 Reflexiones previas sobre el método docente.

2.2 Sistemas de evaluación.

2.3. La evaluación del Trabajo fin de Grado/Trabajo fin de Máster.

\section{Conclusiones.}

IV. Bibliografía.

Enviado: octubre 2019.

Aceptado: diciembre 2019. 


\section{INTRODUCCIÓN}

El proceso de Convergencia Europea que está viviendo la Universidad Española, ha supuesto que se haya modificado el sistema universitario desde una doble perspectiva: de un lado, un cambio estructural con la renovación de las titulaciones y, de otro lado, un cambio metodológico que ha transformado un sistema educativo basado en el autoaprendizaje del alumno. Esto supone, entre otros aspectos, un replanteamiento de las metodologías utilizadas por el profesorado y, por tanto, un cambio en la concepción de su valoración.

De diversa naturaleza y alcance son las consecuencias derivadas de estos procesos de cambio, puesto que centrar la atención en qué, cómo y para qué aprende el alumnado, supone una revolución en la manera tradicional de entender la función docente universitaria. No basta con exigir al estudiante la reproducción de unos contenidos conceptuales, trasmitidos en una clase magistral; por el contrario, el docente universitario deberá capacitar al estudiante para el uso de habilidades o competencias técnicas e intelectuales que le permita acceder, a lo largo de su vida, a un conocimiento que cambia y evoluciona constantemente.

La plasmación en nuestro sistema educativo universitario del Espacio Europeo de Educación Superior -en un periodo de tiempo relativamente corto-, exigió una profunda reflexión, no sólo sobre la estructura y contenidos de los títulos y materias a impartir, sino también una renovación sustancial de las metodologías docentes que venían siendo utilizadas tradicionalmente por el profesorado universitario. El nuevo sistema, ahora centra su interés en el proceso de aprendizaje del alumno, concebido como un proceso dinámico y continuo de conocimientos, habilidades, actitudes y responsabilidades, que han de poder ser evaluadas y medidas para mostrar los resultados de los aprendizajes de un programa educativo.

Los cambios metodológicos que traen consigo los nuevos títulos de Grado y Postgrado vienen acompañados de un modo de evaluar a los estudiantes con una orientación distinta a la tradicional. El sistema de evaluación continua sintoniza 
con esta nueva realidad y, en principio, permite verificar al profesor que se han alcanzado los resultados de aprendizaje relacionados con la adquisición de competencias, previamente determinadas en las memorias de las nuevas titulaciones.

\section{EVALUACIÓN DE COMPETENCIAS}

El proceso general de diseño, implantación y evaluación de los nuevos planes de estudios, centrado en el aprendizaje de competencias, plantea variados interrogantes. Casi ineludiblemente, las primeras cuestiones a las que se ha tratado de dar respuesta se relacionan con qué competencias deben ser adquiridas en una determinada titulación y cómo han de ser demostradas dichas competencias. Por esta razón, en las memorias de los nuevos títulos de grado y posgrado se debieran definir con la mayor claridad posible las competencias, es decir, los conocimientos, las conductas y aptitudes que deben ser adquiridos o puestos en práctica en el ejercicio profesional para el que se capacita con la titulación.

Sin embargo, no es frecuente que se incluyan propuestas que permitan evaluar el logro de los resultados obtenidos en la adquisición de estas competencias. A este respecto, se constata la existencia de un importante vacío respecto a cuánto debe ser observable de la competencia o respecto al dónde ha de reflejarse dicha competencia demostrada. De este modo, elementos que contribuyen a una descripción completa de competencia tales como la manera de constatar el grado de desarrollo de la competencia adquirido por el estudiante y la fuente básica donde comprobar la evidencia de su logro, suelen quedar sin definir.

\subsection{Reflexiones previas sobre el método docente}

Es evidente que la sociedad actual, la situación del mercado de trabajo con elevados índices de desempleo exige, al Sistema de Educación Superior, nuevos retos dirigidos a la formación y preparación de profesionales capaces de afrontar demandas futuras en el ámbito laboral.

A lo largo de estos últimos años, hemos sido testigos de cómo las instituciones de Educación Superior han intentado superar estas dificultades, consiguiéndolo en algunos casos, avanzando en su consecución en otros y, como suele ser habitual, teniendo que hacer frente a nuevas situaciones y a fenómenos emergentes que requerían y, aún requieren, de atención: movilidad estudiantil, caducidad de los conocimientos, formación en competencias, empleo de las TIC, etc. 
Una primera cuestión que se plantea es la de cómo enseñar, es decir las alternativas en el plano de la metodología de la enseñanza para la adquisición de competencias. Y ello porque "de nada serviría la actividad docente si no viene acompañada de la adopción de un planteamiento metodológico en cuanto a la enseñanza de las asignaturas jurídico-laborales, que favorezcan el acceso de los futuros titulados a un conocimiento dinámico y globalizador del Ordenamiento". En este nuevo contexto, aun siendo relevantes los contenidos, siguiendo la filosofía del denominado Proceso de Bolonia, dejan paso a los procedimientos, para asegurar los resultados, adoptando el profesorado el papel de mediador entre el conocimiento y el alumno ${ }^{2}$.

Es importante que el alumno no pierda de vista que está en la Universidad para aprender y no para conseguir un título; es tarea del profesor suscitar en él ese interés por el aprendizaje y por la adquisición de unas competencias que den sentido a su paso por este nivel de enseñanza ${ }^{3}$. El que "me enseñen" deja paso al que "yo aprenda" y el "aprobar" se ve eclipsado por el "aprender", con todo lo que ello implica de modificación de hábitos, metodologías, actitudes, etc. A la vista de estos cambios, se impone una reorientación de las políticas de formación del profesorado ya que, aunque sea evidente, su papel resulta crucial en toda reforma.

En lo que refiere a la enseñanza de materias jurídico-laborales quizá, por los problemas que se le plantean al docente en la enseñanza de esta disciplina jurídica, es trascendente reivindicar un espacio para la reflexión pedagógica propiamente dicha, para el análisis de las dificultades que enfrenta el profesorado a la hora de enseñar y la mejor manera de afrontarlos desde la perspectiva de la didáctica. Y ello porque el docente se forma en la Universidad desde el punto de vista de la materia por enseñar, mientras que la preparación pedagógica queda relegada a la iniciativa personal.

\footnotetext{
${ }^{1}$ SANGUINETTI RAYMOND, W., "La enseñanza del Derecho del trabajo en los estudios de relaciones laborales", en Relaciones Laborales, 1 (1998) 19.

2 Proceso de Bolonia es el nombre que recibe el proceso iniciado a partir de la Declaración de Bolonia en 1999 firmada por países de la UE que no tiene competencias en materia de educación que dio inicio a un proceso de convergencia que tenía como objetivo facilitar el intercambio de titulados y adaptar el contenido de los estudios universitarios a las demandas sociales, mejorando su calidad y competitividad a través de una mayor transparencia y un aprendizaje basado en el estudiante cuantificado a través de los créditos ECTS.

3 PRIETO NAVARRO, L., La enseñanza universitaria centrada en el aprendizaje, Barcelona, Octaedro, 2008, p. 34; SERRANO ARGÜELLO, N., La competencia clave en los estudios de derecho: el razonamiento jurídico. IV Congreso de innovación docente en ciencias jurídicas ISBN: 978-84-694-8683-2.
} 
El fin perseguido con el método docente debe ser el de la transmisión crítica y objetiva de conocimientos propios del saber jurídico-laboral, la facilitación de recursos metodológicos que permita a los alumnos el desarrollo de sus capacidades intelectuales en dicho ámbito, la capacitación para el ejercicio responsable de las actividades profesionales de futuro. Con otras palabras, la docencia universitaria debe proporcionar a quien la recibe un enriquecimiento cultural global donde la labor del docente consistirá, en diseñar metodologías para el logro de la adquisición de las competencias propuestas. Todo ello debe serle puesto de relieve al alumno, animándole en todo momento a la reflexión personal, a la valoración de un mundo jurídico, con el que no siempre ha de estarse de acuerdo y en el que debe aprender a desentrañar el significado verdadero de las pretensiones de la regulación, de las teorías doctrinales y de la fundamentación jurídica de las decisiones judiciales.

En definitiva, la labor docente debe ir dirigida a proporcionar a los alumnos, a partir de un determinado nivel de información suficiente, un conocimiento integral, funcional y crítico de la realidad jurídico-laboral, en definitiva, a enseñarles a aprender.

El concepto de competencias aplicado a las titulaciones jurídico-laborales se relaciona con la necesidad de que el alumnado adquiera unos conocimientos jurídicos básicos que les sirvan para resolver desde un punto de vista legal cada caso concreto, junto a una serie de aptitudes y habilidades fundamentales para el desarrollo de su profesión en el mercado laboral, lo que supondrá sin duda una mayor carga docente. Por tanto, se plantea la necesidad de pasar de las tradicionales clases magistrales a la impartición de una docencia más práctica, en la que el futuro graduado obtenga una formación en habilidades que le sirva como profesional del Derecho.

Es posible observar la competencia desde dos contextos que, aunque diferenciados, están orientados a encontrarse o aproximarse: el mercado laboral y la universidad. La diferencia básica entre el grado de dominio de la competencia que el egresado demuestra en el ejercicio profesional y el que debe adquirir en el marco general de un plan de estudios es que, mientras que en el primer caso este dominio es y debe ser aplicado en una situación real, en el segundo caso se demuestra, en la mayoría de las ocasiones, mediante modelos simulados. La responsabilidad del docente no solamente recae sobre su parcelada tarea de enseñar unos conocimientos o desarrollar unas determinadas habilidades en el estudiante, sino que, también, ha de comprobar y valorar el grado de aprendizaje para asegurar el éxito. 
Desde esta perspectiva integradora del concepto de competencia, el docente debe, no sólo describir, sino también medir el aprendizaje adquirido por el alumno en un ámbito dado y en función de los distintos criterios del contexto que determina la actualización efectiva del saber.

El marco jurídico de los programas universitarios de aprendizaje y evaluación de competencias viene delimitado como es sabido por el RD 1393/2007, de 29 de octubre, por el que se establece la ordenación de las enseñanzas universitarias oficiales. En la citada norma se establecen las directrices para el diseño de los títulos de Grado, Máster y Doctorado.

La concreta referencia a las competencias, la encontramos en el Anexo I, donde se establece que en el apartado tercero de la memoria o propuesta de un título, deberán hacerse constar "las competencias generales y específicas que los estudiantes deben adquirir durante sus estudios y que sean exigibles para otorgar el título», añadiéndose seguidamente y ello es importante, que «las competencias propuestas deben ser evaluables". Por otra parte, en el apartado quinto de la misma memoria o propuesta de título, dedicado a la planificación de las enseñanzas, deberá especificarse entre otras cosas "la descripción de los módulos o materias de enseñanza-aprendizaje que constituyen la estructura del plan de estudios, incluyendo las prácticas externas y el trabajo de fin de Grado o Máster, con los siguientes apartados: a) denominación del módulo o materia; b) competencias que adquiere el estudiante con dicho módulo o materia; c) breve descripción de sus contenidos; d) actividades formativas con su contenido en créditos ECTS, su metodología de enseñanza-aprendizaje y su relación con las competencias que debe adquirir el estudiante; e) sistema de evaluación de la adquisición de las competencias y sistema de calificaciones de acuerdo con la legislación vigente" ${ }^{\wedge}$.

En consecuencia, de la normativa reguladora de las enseñanzas universitarias oficiales, en materia de competencias, se destaca que éstas deben ser evaluables y que la planificación de su aprendizaje y acreditación dentro de cada materia debe hacerse en términos de coherencia.

Junto a las competencias resulta obligado aludir, aunque sea sucintamente, a la distinción entre éstas y los resultados de aprendizaje del alumno, fundamentales en el proceso de evaluación de conocimientos del alumno. En el Marco de cualificaciones del EEES (A Framework for Qualifi cations of the European

\footnotetext{
${ }^{4}$ Real Decreto 1393/2007, de 29 de octubre, por el que se establece la ordenación de las enseñanzas universitarias oficiales. BOE 30 de octubre de 2007. https://www.boe.es/eli/ es/rd/2007/10/29/1393/con.
} 
Higher Education Area) los resultados del aprendizaje son el producto final del proceso de enseñanza, es la adquisición de las competencias a través de un principio esencial de la educación que se aprende lo que se practica, siempre acompañado de retroalimentación y reflexión. El término competencias se utiliza en un sentido amplio permitiendo la gradación de habilidades o destrezas, y se considera que está incluido en el concepto de resultados del aprendizaje. Sin embargo, a lo largo del texto ambos conceptos se utilizan indistintamente, haciendo muchas veces difícil su diferenciación y depende del contexto en que se utilice, porque con frecuencia los resultados del aprendizaje se expresan comúnmente en términos de competencias. Así pues, la línea divisoria entre unos y otras no está siempre clara ${ }^{5}$.

\subsection{Sistemas de evaluación}

Como se ha señalado, uno de los aspectos fundamentales en el diseño de los títulos de grado es el de la coherencia, entre objetivos, competencias, resultados de aprendizaje e instrumentos de evaluación de una titulación. De nada servirían que se incorpore un listado de competencias transversales y específicas en las guías docentes, sin adecuar al mismo tiempo las estrategias de aprendizaje y evaluación a la efectiva adquisición de las mismas.

Las universidades tienen, por consiguiente, un teórico margen de flexibilidad a la hora de diseñar programas de aprendizaje y evaluación de competencias, cuestión ante la que se abren distintas posibilidades organizativas. Así, dichos programas pueden contener decisiones relativas al «quién» y al «cuándo» de la evaluación, buscándose modelos de coordinación a distintos niveles (universidad, titulación, curso y materia), y por otra parte, decisiones que afecten al «cómo» tanto del aprendizaje como de la evaluación de dichas competencias ${ }^{6}$.

La norma UNE 66173 sobre gestión de competencia, diferencia entre las expresiones "evaluación de competencias y valoración de competencias", definiéndolas de tal forma que la primera engloba a la segunda, al concebir la evaluación como diferentes métodos de valoración de las competencias. A su vez, la norma define la valoración de competencias como la "operación que consiste en determinar el estado de las competencias y habilidades de un individuo". Esta identificación de la evaluación como la valoración del logro

\footnotetext{
${ }^{5}$ Véase su distinción en la guía de apoyo para la redacción y puesta en práctica de los resultados del aprendizaje, publicada por ANECA. http://www.aneca.es/Documentos-y-publicaciones/ Otras-guias-y-documentos-de-evaluacion.

${ }^{6}$ MARQUÈS I BANQUÉ, M., "La evaluación de competencias en la Educación Superior: retos e instrumentos", en Revista de Educación y Derecho, 0 (2009) 22-47.
} 
de la competencia adquirida por el estudiante implica, en la práctica, una reorientación del concepto y del proceso de evaluación utilizado en la educación superior, no puede limitarse a determinar lo que un individuo sabe sobre una determinada materia, sino que debe valorar en qué grado el estudiante posee y domina una determinada competencia.

Así configurado, el proceso de evaluación por competencias aparece, esencialmente, como un proceso de recogida, procesamiento y valoración de información, orientado a determinar en qué medida el estudiante ha adquirido el conocimiento y dominio de una determinada competencia o conjunto de competencias. La evaluación debe de constituir una oportunidad de aprendizaje y utilizarse no para adivinar o seleccionar a quien posee ciertas competencias, sino para promoverlas en todos los estudiantes ${ }^{7}$.

Sin embargo, los profesores universitarios todavía tienen dificultades para entender qué es una competencia, cómo se adquiere y cuándo se entiende que se ha adquirido, dificultad que se incrementa cuando se habla de competencias específicas. Clarificar todo lo que se pueda en este sentido no es una cuestión menor, si consideramos que de ello depende la correcta evaluación de los alumnos y su calificación, y lo que es aún más importante, de todo el proceso de enseñanza-aprendizaje.

La pregunta clave de la que debiera partirse para cuestionarnos cómo llevar a cabo la evaluación, sería: ¿Qué es lo que influye más en los alumnos: la docencia o la evaluación? Por consiguiente, la mayoría de las actividades que desarrolla el alumno tiene como objetivo el examen, no el propio proceso de enseñanza y de aprendizaje. El alumno selecciona el volumen y qué estudia en función del examen. Programa su estrategia de tiempo, esfuerzo y dedicación al examen. No presta apenas atención al proceso de aprendizaje. Cambiar esta actitud en los estudiantes va a requerir tiempo y esfuerzo.

La evaluación es un instrumento que viene a cumplir una doble finalidad: facilitar información al profesor sobre la validez de las estrategias didácticas empleadas y su actuación docente y constatar la progresión del aprendizaje o del rendimiento académico del alumno. Precisamente estas dos finalidades se corresponden con el doble aspecto que puede predicarse de todo sistema de evaluación. Si bien es cierto que evaluar supone generalmente obtener información para utilizarla en la confirmación de juicios y en la adopción de decisiones sobre la calificación del alumno, en un sentido más amplio la evaluación en la Universidad tiene un segundo aspecto que refiere a la actuación

7 CANO GARCÍA, E., La evaluación por competencias en la educación Superior. http://www.ugr.es/local/recfpro/rev123COL1. 
docente y que podría catalogarse como un sistema de evaluación del profesor por parte del alumno. Los diseños por competencias permiten aglutinar los saberes tradicionalmente considerados "académicos" con una orientación más "pragmática" que enseñe al alumno/a tomar decisiones, a asumir responsabilidades, a organizar sus tiempos para realizar su trabajo tal y como les va a requerir el ejercicio de la profesión en un futuro inmediato ${ }^{8}$.

Dado que los objetivos de la enseñanza se condicionan a la adquisición de una serie de competencias prefijadas, en la misma medida se estará de acuerdo en que la evaluación del alumno ha de ser personalizada, continua y periódica, a los fines de determinar, su rendimiento, actitud y aprovechamiento.

Dentro de los variados sistemas de evaluación la prueba o examen sigue constituyendo el medio necesario para acreditar el nivel de conocimientos alcanzados. Instrumento que admite, a su vez, diversas variantes, desde las pruebas orales a las escritas, pudiendo transitar ésta últimas desde la exposición de temas largos, a la contestación de preguntas cortas, a la realización de preguntas tipo test $\mathrm{o}$, incluso, a la combinación de preguntas teóricas con la resolución de casos prácticos. Todos estos sistemas poseen ventajas y también algunos inconvenientes, por lo que sería incluso recomendable la posibilidad de su combinación, a los efectos de alcanzar una valoración más adecuada y ajustada del rendimiento del alumno, así como de su proceso de aprendizaje.

La prueba oral tiene sus ventajas permite, sin duda, una mayor ponderación del domino de la materia que tenga el alumno, en la medida en que facilita el intercambio de criterios con él, evita la contestación ajena a la pregunta, permite inquirir por las cuestiones básicas si el alumno no las ha expuesto y, lo más importante, fomenta la iniciación al futuro profesional del Derecho en la técnica de la expresión hablada, que será de la que esencialmente se tenga que servir, al tiempo que agiliza la evaluación de los conocimientos. Además, el examen oral elimina de entrada muchas de las fórmulas de picaresca que tienen las pruebas escritas.

Ahora bien, el examen o prueba oral presenta también inconvenientes, principalmente porque aumenta el subjetivismo del profesor, que debe tomar un mayor distanciamiento respecto de quien se examina, incrementa el margen de error en el alumno debido a que su disposición psicológica resulta más tensa e impide, un adecuado control de resultados porque las revisiones de la calificación son bastante más dificultosas, cuando no imposibles.

${ }^{8}$ LIÑÁN GARCÍA, A., "Metodología de la elaboración de trabajos de investigación jurídica desde un enfoque práctico. El valor de la experiencia profesional", en Revista Jurídica de Investigación e Innovación Educativa, 11 (2015) 81 y ss. 
El examen o prueba escrita en sus diferentes modalidades es el instrumento más frecuentemente utilizado como técnica de evaluación, así como el preferido por la mayoría de los estudiantes, en la creencia de que ésta es la técnica que les ofrece mayores posibilidades e incluso garantías. Tiene a su favor el fomento de la capacidad de síntesis, reflejando claramente la actitud de su estructuración, siendo también reflejo de la destreza del alumno en la expresión escrita; elemento éste también de importancia en el futuro desarrollo de su actividad profesional.

Son diversas las posibilidades que ofrece la prueba escrita, variando desde el desarrollo más o menos amplio de temas del programa, hasta el planteamiento de cuestiones de respuesta de corta extensión, toda vez que la contestación a uno o varios temas del programa cuenta con el gran inconveniente de que, al margen del factor suerte, el resultado de esta modalidad no demuestra que el alumno domine las diferentes partes del programa en su totalidad. Todo ello, sin perjuicio de admitir que la combinación de estas dos modalidades resultaría ser la más aconsejable, pues el alumno puede evidenciar, por una parte, que conoce en su conjunto la materia y, por otra, que tiene capacidad de síntesis y reflexión para afrontar la exposición escrita de un tema en concreto. Las pruebas tipo test tampoco resultan especialmente positivos, en la medida en que, en su mayor parte, desarrollan la agilidad memorística.

En razón de la estrecha conexión que debe existir entre el método docente y el sistema de evaluación y teniendo en cuenta las dimensiones de los grupos de alumnos, al igual que se defiende la combinación entre las clases teóricas y prácticas, se propone que la técnica para evaluar el conocimiento del alumno sea el examen teórico-práctico combinado con una prueba optativa oral. Todo ello, sin perjuicio de otros elementos o criterios objetivos de valoración de que pueda disponer el profesor, tales como trabajos de diferente índole realizados durante el curso, exposiciones orales, y participación en clase. Independientemente de los condicionamientos que la realidad pueda imponer en cada caso -léase, dimensión del grupo de alumnos-, lo cierto es que la técnica de evaluación de una asignatura viene condicionada por la forma en la que esta última ha sido impartida por el profesor a lo largo del curso.

Por tanto, en la técnica evaluadora óptima parece imprescindible que la calificación del alumno gire en torno a una combinada relación de cuestiones de naturaleza puramente teórica y la resolución de un supuesto práctico en donde se demuestre su capacidad de análisis jurídico, esto es, que realmente ha comprendido aquello que ha estudiado. El tipo de prueba evaluadora de la adquisición de competencias que resulta más acorde con los objetivos anteriormente planteados en la tarea docente es el planteamiento de una 
prueba escrita teórico-práctica, con cuestiones primordialmente cortas que exijan abordar y razonar cuestiones esenciales de la asignatura, con el fin de comprobar el aprendizaje del alumno para la adquisición de materias, su expresión jurídica y en última instancia su capacidad de síntesis dentro de la amplitud y extensión de la disciplina.

En cualquier caso, al margen de la idoneidad de las técnicas evaluadoras, resulta obligado insistir en la formación del profesorado, siendo su meta última la de promover docentes universitarios capaces de enseñar a aprender y hacer pensar a sus estudiantes. En esta misma línea cabe incidir en la necesidad de que las políticas institucionales den un giro en favor de la potenciación de la docencia. El esfuerzo para el docente podría venir representado, en primer lugar, por la planificación, con la necesidad de seleccionar pruebas, establecer criterios de corrección o fijar un calendario de realización; y prosigue, en segundo término, con el desarrollo, que exige preparar y corregir las distintas pruebas, al margen de mantener al alumno constantemente informado de su evolución.

Por su parte, también sería necesario analizar el cambio de las técnicas y métodos docentes desde la perspectiva del alumno admiten el cambio y donde habría que dedicar especial atención a la dificultad que para el estudiante supone la autonomía que se le da ahora para autoprogramarse, algo a lo que no están demasiado acostumbrados.

\subsection{La evaluación del Trabajo Fin de Grado/Trabajo Fin de Máster}

Desde el año 2010 las Universidades españolas tienen implantados los estudios de grado, respondiendo así a la demanda de crear un espacio europeo de educación superior. Bajo esta regulación todas las enseñanzas universitarias, sean titulaciones de Grado o Master, han de concluir con la elaboración y defensa de un trabajo de fin de grado (TFG) o trabajo fin de máster (TFM).

El Trabajo Fin de Grado tendrá un mínimo de 6 créditos y un máximo del 12,5 por ciento del total de los créditos del título y que deberá realizarse en la fase final del plan de estudios y estar orientado a la evaluación de competencias asociadas al título9. Los Trabajos fin de Máster deberán concluir “con la elaboración y defensa pública de un trabajo de fin de Máster, que tendrá entre 6 y 30 créditos" 10 .

9 Art. 12.7 del Real Decreto 1393/2007, de 29 de octubre, por el que se establece la ordenación de las enseñanzas universitarias oficiales[consultado 14 Nov 2019]. Disponible en: https://www.boe.es/buscar/pdf/2007/BOE-A-2007-18770-consolidado.pdf.

${ }^{10}$ Art. 15.3 del Real Decreto 1393/2007, de 29 de octubre, por el que se establece la ordenación de las enseñanzas universitarias oficiales. 
Sobre estas premisas legales los distintos planes de estudio de las titulaciones jurídicas han hecho propuestas para el desarrollo del TFG/TFM. No obstante, se han encontrado diversos inconvenientes, principalmente, relacionados con la defensa y evaluación del TFG/TFM.

De la lectura de los preceptos se deduce una mayor exigencia en el TFM en relación con el TFG, justificado por el nivel avanzado de conocimiento que debe contemplarse en un título de Máster. La alusión única a "defensa" es indeterminada y ha generado confusión sobre su aplicación, ¿exige un tribunal?, ¿puede realizarse grupalmente?... Estas y otras cuestiones han intentado resolverse por las agencias de calidad colaboradoras de la ANECA, en las distintas Comunidades Autónomas.

En el caso de las universidades madrileñas, la normativa reguladora de los estudios universitarios se ha desarrollado en una guía de ayuda al diseño de planes de estudio de Grado y Máster, realizada por La Fundación para el Conocimiento Madri $+d$ que es el órgano de evaluación en el ámbito universitario de la Comunidad de Madrid ${ }^{11}$.

En el citado documento se establecen como premisas básicas para la válida constitución, funcionamiento y posterior evaluación de los TFG/TFM que deberán definirse de forma independiente, estar bien diferenciado del resto de módulos o materias y tener entidad propia. La realización de TFG/TFM constituye una actividad formativa que debe contemplarse en el cómputo de los ECTS del plan de estudios. Aunque el TFG/TFM se realice en equipo, debe defenderse y evaluarse de manera individual. La defensa de los TFM será pública, ante un tribunal universitario. La defensa del TFM puede realizarse mediante videoconferencia en cualquiera de sus modalidades, siempre que: 1) Una persona en representación de la universidad asegure presencialmente la identidad del estudiante en el lugar donde éste realice la defensa del TFM y le acompañe durante la misma; 2) La defensa sea pública, bien donde esté presente el estudiante o bien donde esté presente el tribunal; 3) Exista posibilidad de interacción entre el estudiante y el tribuna $1^{12}$.

Las universidades españolas, con el fin de unificar los criterios y procedimientos en la organización y evaluación del TFG/TFM, han desarrollado marcos reguladores de carácter interno. La mayoría de ellos contienen: definición y tipologías del TFG, organización, procedimiento de elaboración, sistema de tutorización, requisitos de presentación y modalidades de evaluación. Aun y

\footnotetext{
${ }^{11}$ Decreto 63/2014, de 29 de mayo, el Consejo de Gobierno de la Comunidad de Madrid

$12 \mathrm{http} / / /$ www.madrimasd.org/uploads/guia_verificacion_v3_sep_2019_iv.pdf.
} 
así, no son pocas las dificultades expresadas entre el profesorado en lo que respecta a la dirección y tutela de esta asignatura, abarcando desde la elección del tipo de trabajo hasta la preparación para su exposición y defensa.

De lo que no hay duda es que se trata de la asignatura final de los estudios de Grado o Máster, en la medida en que su propósito principal es poner de manifiesto que se han alcanzado las competencias vinculadas a la titulación. Además, la realización del TFG/TFM va a permitir que se desarrollen competencias específicas y propias de la formación universitaria e imprescindibles para los profesionales que necesita la sociedad, como son la capacidad de gestionar la información, de razonamiento crítico y de resolución de problemas.

Para garantizar la eficacia en la adquisición de competencias en el TFG/TFM, resulta fundamental la labor desempeñada por el tutor en elementos como la elección del tipo de trabajo, la estructura y desarrollo de este, su redacción científica y las técnicas de defensa y exposición eficaces. La figura del tutor es esencial, debe ser facilitadora del aprendizaje a través de un debate y negociación continuos con el alumno en todas las fases del TFG/TFM (selección del tema, plan de trabajo, desarrollo, evaluación y presentación pública). A este respecto uno de los condicionantes principales que pudieran limitar esta eficacia reside en la universidad pública en el número de alumnos para tutorizar que tenga asignados cada profesor ${ }^{13}$.

\section{CONCLUSIONES}

Clarificar el contenido de las competencias específicas y fijar indicadores es un objetivo aún no logrado, y su consecución resulta esencial en la medida en que facilitará la adecuación de las metodologías y los mecanismos de evaluación del propio proceso de enseñanza o aprendizaje, y servirá como herramienta para la detección de posibles carencias.

Los listados de competencias en los Planes de estudios de las titulaciones jurídico-laborales siguen siendo, por regla general, muy amplios, repetitivos e incluso, a veces, de difícil adquisición, debiendo colocarse en su vértice el razonamiento jurídico, la programación y desarrollo de las clases, como eje en la evaluación de los alumnos.

${ }^{13}$ SERRANO-GALLARDO, P.; MARTÍNEZ-MARTÍN, Ma.L., y MARTÍNEZ-MARCOS, M., "Factores que determinan la evaluación del trabajo fin de grado. Un análisis multinivel", en Educación Médica, Vol. 19, Issue 6 (November-December 2018) 339-347. 
Se han omitido en las memorias de los títulos y en las guías de las asignaturas cómo se van a desarrollar y, en ocasiones, resulta complejo evaluar dichas competencias. No se ha tenido en cuenta la propia transversalidad de dichas competencias en el espacio (varias asignaturas del mismo semestre) y en el tiempo (varias asignaturas de diferentes semestres). En este sentido se percibe una falta de coordinación entre las asignaturas que van a trabajar una misma competencia, parece lógico que cuando hablamos de evaluación de competencias deba buscarse algún mecanismo que a la práctica evite que un profesor pueda, por ejemplo, decidir que un estudiante sabe utilizar principios jurídicos y valores fundamentales como instrumento de trabajo en la interpretación del ordenamiento jurídico1, al mismo tiempo que otro profesor, a la vista de las actividades realizadas en su materia, decida unilateralmente justamente lo contrario $^{14}$

A ello, habrá que sumar el dato de que, a pesar de las buenas intenciones manifestadas, no siempre las políticas educativas seguidas contribuyen a dotar a los profesores del apoyo e incentivos necesarios a su transcendental tarea. Por tanto, la generosidad, el buen hacer y el esfuerzo continuado y coordinado en la innovación de toda la comunidad universitaria podrán lograr a corto plazo resultados positivos que, al hacerse visibles se conviertan en un incentivo para seguir avanzando.

En definitiva, partiendo del convencimiento de que no existe un sistema de evaluación del alumno que sea perfecto, el cambio está fundamentalmente en el "cómo" se pueden aprender los contenidos (en un contexto donde la información es fácilmente accesible) y afecta a la planificación, a la metodología y a la evaluación. Se requieren nuevos marcos de trabajo, colegiados, donde tenga cabida el desarrollo de experiencias de aprendizaje atractivas e integradas. $Y$ todo ello ha de llevar aparejado un cambio en la evaluación en la línea de lo ya señalado. Si se cambia la evaluación (no sólo la instrumentación y los agentes implicados, sino su propia lógica y la finalidad), probablemente cambie todo el proceso. Se pasará de una evaluación de los aprendizajes a una evaluación para los aprendizajes y debe marcarse como principal objetivo que toda reforma educativa debiera buscar: la calidad y el aprendizaje del alumno para afrontar su futuro profesional.

${ }^{14}$ MARQUÈS I BANQUÉ, M., "La evaluación de competencias en la Educación Superior: retos e instrumentos", en Revista de Educación y Derecho, 0 (2009) 22-47. 


\section{BIBLIOGRAFÍA}

- AAVV, Enseñar Derecho en el siglo XXI. Una guía práctica sobre el Grado en Derecho, en PALOMINO LOZANO, R. (Dir.), Pamplona, Aranzadi/ Thomson, 2009.

- BELLOSO MARTÍN, N., "La construcción del espacio europeo de educación superior -EEES- en las universidades españolas: la metodología docente y el proceso de evaluación del aprendizaje del alumno en los estudios jurídicos", en Revista seqüencia, 59 (2009).

- CANO GARCÍA, E., "La evaluación por competencias en la educación Superior": http://www.ugr.es/local/recfpro/rev123COL1.pdf.

- LIÑÁN GARCÍA, A., "Metodología de la elaboración de trabajos de investigación jurídica desde un enfoque práctico. El valor de la experiencia profesional", en Revista Jurídica de Investigación e Innovación Educativa, 11 (2015).

- MARQUÈS I BANQUÉ, M., "La evaluación de competencias en la Educación Superior: retos e instrumentos", en Revista de Educación y Derecho, 0 (2009) $22-47$.

- PRIETO NAVARRO, L., La enseñanza universitaria centrada en el aprendizaje, Barcelona, Octaedro, 2008.

- SANGUINETTI RAYMOND, W., "La enseñanza del Derecho del trabajo en los estudios de relaciones laborales", en Relaciones Laborales, 1 (1998).

- SERRANO ARGÜELLO, N., La competencia clave en los estudios de derecho: el razonamiento jurídico. IV Congreso de innovación docente en ciencias jurídicas ISBN: 978-84-694-8683-2.

- SERRANO-GALLARDO, P.; MARTÍNEZ-MARTÍN, Mª.L., y MARTÍNEZMARCOS, M., "Factores que determinan la evaluación del trabajo fin de grado. Un análisis multinivel", en Educación Médica, Vol. 19, Issue 6 (November-December 2018) 339-347. 